\title{
Soluble Lectin-Like Oxidized Low-Density Lipoprotein Receptor-1 as an Early Biomarker for ST Elevation Myocardial Infarction - Time-Dependent Comparison With Other Biomarkers -
}

\author{
Nobuaki Kobayashi, MD; Noritake Hata, MD; Noriaki Kume, MD; Yoshihiko Seino, MD; \\ Toru Inami, MD; Shinya Yokoyama, MD; Takuro Shinada, MD; Kazunori Tomita, MD; \\ Toshihiko Kaneshige, PhD; Kyoichi Mizuno, MD
}

\begin{abstract}
Background: The diagnostic sensitivity of myocardial necrosis markers, such as creatine kinase-MB (CK-MB), cardiac troponins, myoglobin and heart-type fatty acid-binding protein (H-FABP) for the earliest stage of STelevation myocardial infarction (STEMI), remains insufficient. We compared a new biomarker of plaque vulnerability (soluble lectin-like oxidized low-density lipoprotein receptor-1, sLOX-1) with other biomarkers at the earliest stage of STEMI.

Methods and Results: Plasma sLOX-1 levels were measured in 125 STEMI, 44 non-STEMI (NSTEMI) and 125 non-acute myocardial infarction (non-AMI) patients and were significantly $(P<0.0001)$ higher in the STEMI and NSTEMI than in the non-AMI patients (median, 25th and 75th percentiles: 241.0, 132.3 and 472.2 vs. $147.3,92.9$ and 262.4 vs. $64.3,54.4$ and $84.3 \mathrm{pg} / \mathrm{ml}$, respectively). At the optimal cut-off value of $91.0 \mathrm{pg} / \mathrm{ml}$, sLOX-1 discriminated STEMI from non-AMI with $89.6 \%$ sensitivity and $82.4 \%$ specificity. Time-dependent changes in sLOX-1, $\mathrm{H}-\mathrm{FABP}$, myoglobin, troponin T and CK-MB were analyzed in 27 STEMI patients. Elevated plasma sLOX-1 levels persisted for $24 \mathrm{~h}$ after admission, whereas other markers were not elevated at the time of admission and peaked at $\geq 2 \mathrm{~h}$ thereafter. The diagnostic sensitivity of sLOX-1, H-FABP, myoglobin, troponin T and CK-MB for STEMI upon admission (89 min after onset) was $93 \%, 78 \%, 70 \%, 56 \%$ and $33 \%$, respectively.
\end{abstract}

Conclusions: Plasma sLOX-1 diagnosed the early stages of STEMI more accurately than H-FABP, myoglobin, troponin T and CK-MB. (Circ J 2011; 75: 1433-1439)

Key Words: Diagnostic predictor; Ischemic injury; Myocardial necrosis; Plaque rupture

A lthough cardiovascular research and medicine are continuing to advance, ${ }^{1}$ and the diagnosis and treatment of acute myocardial infarction (AMI) have significantly progressed over the past decade, AMI remains a major cause of mortality in developed countries. ${ }^{2}$ Early re-opening of an infarct-related coronary artery using fibrinolysis or percutaneous coronary intervention (PCI) is the most important strategy for treating ST-elevation myocardial infarction (STEMI). ${ }^{3,4}$ Early diagnosis of STEMI is critical for successful implementation of the early-open-artery theory.,5

AMI is currently diagnosed using biomarkers of myocar- dial damage, such as creatine kinase-MB (CK-MB), ${ }^{6}$ cardiac troponins, ${ }^{7-11}$ myoglobin ${ }^{12}$ and heart-type fatty acid-binding protein (H-FABP), ${ }^{13}$ each of which has advantages and disadvantages. ${ }^{14}$ Although CK-MB and cardiac troponin ${ }^{15,16}$ are the previous and present gold standard markers, respectively, for myocardial necrosis, neither is an early marker of myocardial necrosis because they only start to elevate $3-4 \mathrm{~h}$ after the onset of AMI. On the other hand, although myoglobin and $\mathrm{H}-\mathrm{FABP}$ are reported to be useful for the early detection of myocardial damage, their diagnostic specificity for AMI is insufficient. ${ }^{12,13}$

Received September 8, 2010; revised manuscript received December 14, 2010; accepted January 24, 2011; released online March 30, 2011 Time for primary review: 28 days

Division of Intensive Care Unit, Chiba Hokusoh Hospital, Nippon Medical School, Chiba (N. Kobayashi, N.H., S.Y., T.S., K.T.); Department of Cardiovascular Medicine, Graduate School of Medicine, Kyoto University, Kyoto (N. Kume); Department of Internal Medicine, Chiba Hokusoh Hospital, Nippon Medical School, Chiba (Y.S., T.I.); Diagnostics Division, Shionogi \& Co. Ltd, Osaka (T.K.); and Division of Cardiology, Hepatology, Geriatrics and Integrated Medicine, Department of Internal Medicine, Nippon Medical School, Tokyo (K.M.), Japan

Mailing address: Nobuaki Kobayashi, MD, Division of Intensive Care Unit, Chiba Hokusoh Hospital, Nippon Medical School, 1715 Kamagari, Inzai, Chiba 270-1694, Japan. E-mail: s5047@nms.ac.jp

ISSN-1346-9843 doi:10.1253/circj.CJ-10-0913

All rights are reserved to the Japanese Circulation Society. For permissions, please e-mail: cj@j-circ.or.jp 


\section{Editorial $p 1322$}

Plaque instability or rupture followed by thrombus formation precedes cardiac necrosis or ischemic injury in acute coronary syndrome (ACS). ${ }^{17}$ Thus, the identification of biomarkers that can detect plaque instability or rupture might enable earlier diagnosis of AMI. Lectin-like oxidized lowdensity lipoprotein (LDL) receptor-1 (LOX-1) is a receptor for atherogenic oxidized LDL in advanced human atherosclerotic plaques, ${ }^{18}$ and cleaved at the membrane-proximal extracellular domain by proteases into a soluble form. ${ }^{19,20}$ Thus, soluble LOX-1 (sLOX-1) is regarded as a marker of plaque rupture. In our previous studies, sensitive and specific immunoassays for human sLOX-1 revealed its diagnostic value for ACS at the earliest stage. ${ }^{19,21-23}$ Although sLOX-1 is considered to be a useful biomarker for the early diagnosis of ACS, including STEMI, its time-dependent diagnostic sensitivity for STEMI has not been compared to that of current biomarkers, namely CK-MB, troponin T, H-FABP and myoglobin.

\section{Methods}

We planned two studies to clarify the value of SLOX-1 for diagnosing STEMI and non-STEMI (NSTEMI) (Study-1), and the time-dependent changes of the sLOX-1 level in the early stages of STEMI (Study-2). The ethics committee of the hospital approved the study protocols, and written informed consent to participate in the investigations was given by all the study patients.

\section{Study Population}

Study-1 Between October 2007 and December 2009 at Nippon Medical School Chiba-Hokusoh Hospital we assigned consecutive 125 STEMI patients (111 males, 14 females; mean age: $64 \pm 12$ years) and 44 NSTEMI patients (30 males, 14 females; mean age: $66 \pm 10$ years) who arrived at the emergency room (ER) within $24 \mathrm{~h}$ of the onset of chest symptoms and underwent coronary angiography (CAG). In addition, between October and December 2007 we assigned consecutive 125 patients ( 80 males, 45 females; mean age: $66 \pm 11$ years) who underwent CAG without AMI (non-AMI group; 98 stable coronary artery disease (CAD) and 27 non-CAD) as the reference group.

Study-2 From October 2008 to February 2009 at Nippon Medical School Chiba-Hokusoh Hospital we enrolled 27 consecutive patients with STEMI (25 males, 2 females; mean age: $63 \pm 13$ years) who arrived at the ER within $5 \mathrm{~h}$ of the onset of chest symptoms and underwent CAG. We excluded the NSTEMI population from this study because there were many cases in which the exact time of onset was unclear because of the recurrent or ambiguous chest symptoms in the NSTEMI population.

In both studies, AMI was defined as prolonged chest pain ( $\geq 30 \mathrm{~min}$ ) with the coronary artery lesion evidenced by CAG. STEMI and NSTEMI were respectively defined as AMI with and without ST segment elevation $(\geq 0.2 \mathrm{mV})$ in at least 2 contiguous leads on ECG. Patients undergoing renal replacement therapy for acute or chronic kidney disease and those on a percutaneous cardiopulmonary support system for cardiogenic shock or fatal arrhythmia were excluded.

Measurement of SLOX-1 and Other Plasma Biomarkers

Sampling Methods Study-1: Peripheral blood samples were obtained immediately before starting cardiac catheterization, and plasma separated by centrifugation was stored frozen at $-80^{\circ} \mathrm{C}$. Study-2: Peripheral blood samples were collected from the radial or femoral artery of each patient at the following time points: arrival at the ER (point 1), immediately before starting CAG (point 2), immediately after terminating PCI (point 3), at 6 (point 4), 12 (point 5) and 24 (point 6) hours after arrival at the ER and on discharge from the hospital (point 7).

Biomarker Measurements Plasma sLOX-1 levels were measured using a sandwich chemiluminescent enzyme immunoassay (CLEIA) with 2 newly developed anti-human LOX-1 monoclonal antibodies, ${ }^{23}$ which was modified from the previously described enzyme-linked immunosorbent assay (ELISA) by use of the former polyclonal antibodies. ${ }^{22} \mathrm{CK}-\mathrm{MB}$, troponin $\mathrm{T}$, myoglobin and $\mathrm{H}-\mathrm{FABP}$ were measured using a chemiluminescent enzyme immunoassay (ADVIA Centaur; Siemens Healthcare Diagnostics Inc, Tokyo, Japan), an electrochemiluminescence immunoassay (ECLusys Troponin TIII; Roche Diagnostics Co, Tokyo, Japan), a double antibody radioimmunoassay (Myoglobin Kit "Daiichi" III; TFB Inc, Tokyo, Japan) and an enzyme-linked immunosorbent assay (Markit-M H-FABP; DS Pharma Biomedical Co Ltd, Osaka, Japan), respectively.

\section{Statistical Analysis}

Continuous variables are presented as mean \pm standard deviation and were compared using 1-way ANOVA test. Dichotomous variables were compared using $\chi^{2}$ statistics. Because sLOX-1 levels and the time intervals did not distribute normally, these values are presented as median, and 25th and 75th percentiles and were compared between 2 groups by MannWhitney U test. Multivariable logistic regression analysis was applied to identify factors that were independently associated with AMI, by inclusion of factors that were significantly $(\mathrm{P}<0.05)$ associated with AMI in the univariable analysis. The optimal cut-off values of plasma sLOX-1 to diagnose STEMI and NSTEMI were calculated from a receiver-operating characteristic (ROC) curve generated from Study-1. The diagnostic sensitivity of the biomarkers for STEMI were calculated in Study-2 using this cut-off value of SLOX-1 and the known cut-off values of the other biomarkers at each sampling point. Data were statistically analyzed using the SPSS software package, version 16.0. A P-value $<0.05$ was considered statistically significant.

\section{Results}

\section{Comparison of sLOX-1 Levels in STEMI, NSTEMI and Non-AMI Patients (Study-1)}

The characteristics of the enrolled patients are shown in Table 1. Age, prevalence of risk factors, such as diabetes, hypertension, dyslipidemia, familial history of AMI and smoking, and serum creatinine levels were comparable among the STEMI, NSTEMI and non-AMI groups. Male sex and smokers were significantly more prevalent in the STEMI than in the NSTEMI and non-AMI groups; however, their prevalences were not significantly different between NSTEMI and non-AMI. Peak serum CK-MB levels were significantly higher in STEMI than in NSTEMI patients $(\mathrm{P}<$ 0.001). The culprit vessels in AMI were comparable between STEMI and NSTEMI patients, except that left circumflex lesions were more prevalent in NSTEMI than in STEMI patients. The number of diseased vessels was comparable in both AMI groups. In addition, there was no significant cor- 


\begin{tabular}{|c|c|c|c|c|}
\hline & \multicolumn{2}{|c|}{ AMI } & \multirow{2}{*}{ Non-AMI $(n=125)$} & \multirow{2}{*}{ P value* } \\
\hline & STEMI $(n=125)$ & NSTEMI $(n=44)$ & & \\
\hline Male, n (\%) & $111(89)^{\star, \star *}$ & $14(68)^{*}$ & $80(64)^{\star *}$ & $<0.001$ \\
\hline Age (years), mean $\pm S D$ & $64 \pm 12$ & $66 \pm 10$ & $66 \pm 11$ & 0.430 \\
\hline \multicolumn{5}{|l|}{ Risk factors, n (\%) } \\
\hline Diabetes & $45(36)$ & $11(25)$ & $37(30)$ & 0.327 \\
\hline Hypertension & $75(60)$ & $34(77)$ & $87(70)$ & 0.074 \\
\hline Dyslipidemia & $79(63)$ & $30(68)$ & $89(71)$ & 0.399 \\
\hline Familial history of AMI & $24(19)$ & $11(25)$ & $37(30)$ & 0.160 \\
\hline Smoking & $83(66)^{t, t \dagger}$ & $21(48)^{\dagger}$ & $47(38)^{\dagger \dagger}$ & $<0.001$ \\
\hline Creatinine $(\mathrm{mg} / \mathrm{dl})$, mean $\pm \mathrm{SD}$ & $0.90 \pm 0.23$ & $0.90 \pm 0.48$ & $0.92 \pm 0.36$ & 0.905 \\
\hline Peak CK-MB (IU/L), mean \pm SD & $177 \pm 199$ & $43 \pm 94$ & & $<0.001$ \\
\hline \multicolumn{5}{|l|}{ Culprit vessel, n (\%) } \\
\hline Right coronary artery & $48(38)$ & $10(23)$ & & 0.060 \\
\hline Left anterior descending artery & $65(52)$ & $24(55)$ & & 0.771 \\
\hline Left circumflex artery & $12(10)$ & $10(23)$ & & 0.026 \\
\hline No. of diseased vessels, mean \pm SD & $1.76 \pm 0.81$ & $1.71 \pm 0.77$ & & 0.692 \\
\hline
\end{tabular}

AMI, acute myocardial infarction; STEMI, ST elevation myocardial infarction; NSTEMI, non-ST elevation myocardial infarction; SD, standard deviation; CK, creatine kinase.

Continuous variables were compared by 1-way ANOVA and by Student's t-test, among STEMI, NSTEMI and nonAMI groups, and between STEMI and NSTEMI groups, respectively. Dichotomous variables were compared among 3 groups by $\chi^{2}$ test.

${ }^{*} \mathrm{P}=0.002$ between STEMI and NSTEMI by $\chi^{2}$ test; ${ }^{* *} \mathrm{P}<0.001$ between STEMI and non-AMI by $\chi^{2}$ test; ${ }^{\dagger} \mathrm{P}=0.029$ between STEMI and NSTEMI by $\chi^{2}$ test; ${ }^{\mathrm{T}} \mathrm{P}<0.001$ between STEMI and non-AMI by $\chi^{2}$ test.

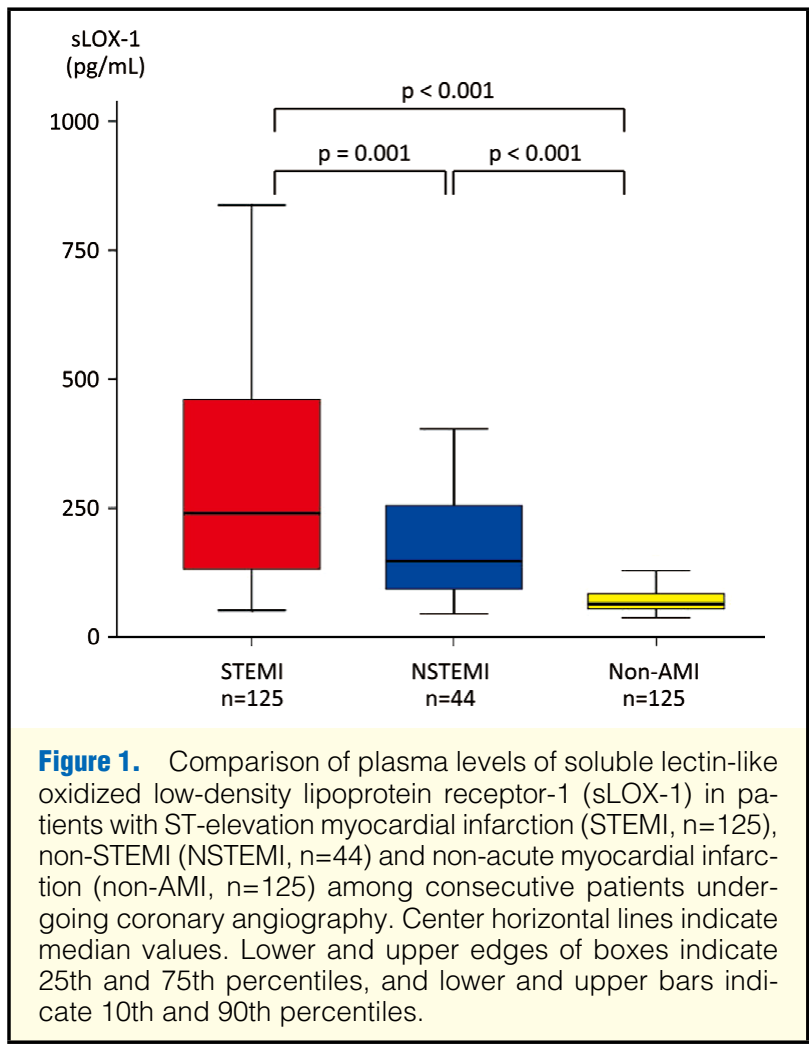

relation between plasma SLOX-1 and serum creatinine levels $(\mathrm{P}=0.203)$, indicating that renal function may not significantly affect plasma sLOX-1 levels. As shown in Figure 1, plasma levels of sLOX-1 were significantly higher in the STEMI $(\mathrm{P}<$ $0.001)$ and NSTEMI $(\mathrm{P}<0.001)$ groups than in the non-AMI group. In STEMI patients, plasma sLOX-1 levels were sig-

\begin{tabular}{|lrcc|}
\hline Table 2. Comparison of sLOX-1 Levels in Patients With and \\
Without Risk Factors \\
Male & $\mathbf{n}$ & $\begin{array}{c}\text { sLOX-1 (pg/ml), } \\
\text { mean } \pm \text { SD }\end{array}$ & P value \\
Female & 221 & $228.4 \pm 269.7$ & $<0.001$ \\
Familial history of AMI, (+) & 73 & $145.6 \pm 213.4$ & \\
Familial history of AMI, (-) & 72 & $173.0 \pm 225.1$ & 0.054 \\
Hypertension, (+) & 222 & $219.1 \pm 268.8$ & \\
Hypertension, (-) & 196 & $198.9 \pm 246.5$ & 0.370 \\
Diabetes, (+) & 98 & $225.8 \pm 282.2$ & \\
Diabetes, (-) & 93 & $205.6 \pm 183.2$ & 0.077 \\
Dyslipidemia, (+) & 201 & $208.9 \pm 288.0$ & \\
Dyslipidemia, (-) & 198 & $210.4 \pm 260.1$ & 0.790 \\
Smoking, (+) & 96 & $202.6 \pm 258.4$ & \\
Smoking, (-) & 151 & $254.4 \pm 287.4$ & $<0.001$ \\
\hline
\end{tabular}

sLOX-1, soluble lectin like oxidized low-density lipoprotein receptor-1. Other abbreviations see in Table 1.

nificantly more elevated than in NSTEMI patients $(\mathrm{P}=0.001)$. Median and 25th and 75th percentile values for STEMI, NSTEMI and non-AMI were 241.0, 132.3 and 472.2 vs. $147.3,92.9$ and 262.4 vs. $64.3,54.4$ and $84.3 \mathrm{pg} / \mathrm{ml}$, respectively. In AMI patients, the number of diseased coronary vessels did not significantly affect plasma sLOX-1 levels; median sLOX-1 values for 1,2 and 3 diseased vessels were $171.4,214.1$ and $245.7 \mathrm{pg} / \mathrm{ml}$, respectively $(\mathrm{P}=0.466)$. In addition, plasma sLOX-1 levels correlated poorly with peak serum CK-MB levels (Spearman $\rho=0.387, \mathrm{P}<0.001$ ). Plasma levels of sLOX-1 did not significantly differ between those with and without risk factors, such as a family history of AMI, hypertension, diabetes or dyslipidemia, except that sLOX-1 levels were significantly higher in males and smokers than in females and non-smokers (Table 2). Although males and smokers 


\begin{tabular}{lccc|}
\hline Table 3. Multivariable Logistic Regression Analysis to \\
Identify Factors Associated With AMI
\end{tabular}

$\mathrm{OR}$, odds ratio; $\mathrm{Cl}$, confidence interval. Other abbreviations see in Tables 1,2.

were significantly more prevalent in the AMI group than in non-AMI and the sLOX-1 levels were significantly higher in males and smokers, multivariable logistic regression analysis to identify factors independently associated with AMI, including sLOX-1, sex and smoking, revealed that sLOX-1 alone was an independent factor associated with AMI (Table 3).

\section{Defining the Optimal Cut-0ff Value of sLOX-1 for STEMI (Study-1)}

To obtain the optimal cut-off value of sLOX-1 to diagnose STEMI, plasma sLOX-1 levels were compared between the STEMI and non-AMI groups. The ROC curve of sLOX-1 for detecting STEMI using the non-AMI group as a negative reference (Figure 2A) indicated an area under the curve (AUC) value of 0.942 . In addition, this ROC curve analysis revealed that SLOX-1 detected STEMI with $89.6 \%$ sensitivity and $82.4 \%$ specificity at an optimal cut-off value of $91.0 \mathrm{ng} / \mathrm{ml}$. On the other hand, the ROC curve of sLOX-1 for detecting NSTEMI using the non-AMI group as a negative reference (Figure 2B) indicated an AUC value of 0.858 , and sLOX-1 detected NSTEMI with $79.5 \%$ sensitivity and $82.4 \%$ specificity at an optimal cut-off value of $90.3 \mathrm{ng} / \mathrm{ml}$, which was almost identical to the value for STEMI.

\section{Comparison of Time-Dependent Changes in SLOX-1 With Other Biomarkers (Study-2)}

The characteristics of the patients enrolled in the Study-2 are summarized in Table 4. The respective interval (median, 25th and 75th percentiles) from symptom onset to arrival at the ER (point 1) was 89, 66 and $173 \mathrm{~min}$, to the start of CAG (point 2) was 142, 100 and $224 \mathrm{~min}$ and to the termination of PCI (point 3) was 230, 172 and $320 \mathrm{~min}$. Sampling point 7 (discharge) was 16, 12 and 20 days after the onset of STEMI.

The time-dependent changes in sLOX-1, H-FABP, myoglobin, troponin T and CK-MB are shown in Figure 3. High plasma levels of sLOX-1 persisted for $24 \mathrm{~h}$ (point 6) after arrival at the ER (point 1), whereas H-FABP and myoglobin levels peaked at PCI termination (point 3) and then gradually decreased. Troponin $\mathrm{T}$ and CK-MB levels were highest at $6 \mathrm{~h}$ after ER arrival (point 4) and then gradually declined. The time-dependent changes in the sensitivity of each biomarker to diagnose STEMI are shown in Figure 4. The $91.0 \mathrm{pg} / \mathrm{ml}$ cut-off value of sLOX-1 was determined from the ROC curve in Study-1. The cut-off value for H-FABP, myoglobin, tropo$\operatorname{nin} \mathrm{T}$ and CK-MB was $6.2,60,0.02$ and $10.0 \mathrm{ng} / \mathrm{ml}$, respectively, which are the standard cut-off values used to clinically diagnose STEMI in Japan. The sensitivity of sLOX-1 to diagnose STEMI upon arrival at the ER (point 1) was 93\%, compared to $78 \%, 70 \%, 56 \%$ and $33 \%$, for H-FABP, myoglobin, troponin $\mathrm{T}$ and $\mathrm{CK}-\mathrm{MB}$, respectively, indicating a higher diagnostic sensitivity of sLOX-1. These values were $100 \%, 93 \%$, $89 \%, 70 \%$ and $48 \%$, respectively, at the start of CAG (point 2), and $100 \%, 93 \%, 93 \%, 96 \%$ and $89 \%$, respectively, at the termination of PCI (point 3). The diagnostic sensitivity of these biomarkers remained high (89-100\%) at $12 \mathrm{~h}$ after arrival at the ER (point 5). At $24 \mathrm{~h}$ after ER arrival (point 6), the diagnostic sensitivity of sLOX-1, troponin T and CK-MB remained at $89 \%, 100 \%$ and $93 \%$, respectively, whereas those of H-FABP and myoglobin fell to $56 \%$ and $67 \%$, respectively.
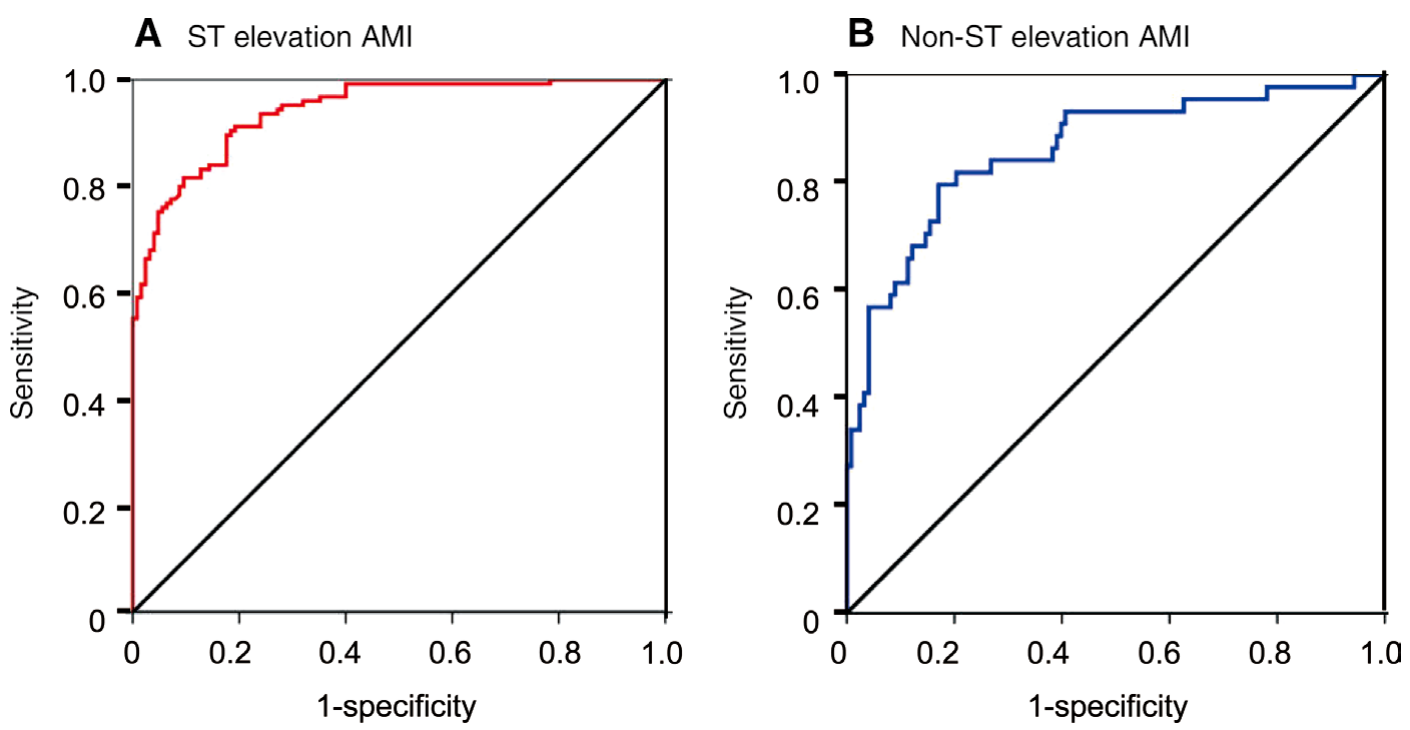

Figure 2. Diagnostic sensitivity and specificity of soluble lectin-like oxidized low-density lipoprotein receptor-1 (sLOX-1) for ST-elevation myocardial infarction (STEMI) and non-STEMI (NSTEMI). Receiver-operating characteristic curves of sLOX-1 for diagnosis of STEMI (A) and NSTEMI (B) are shown. The area under the curve value for sLOX-1 was 0.942 for STEMI, and 0.858 for NSTEMI. Diagnostic sensitivity and specificity for STEMI were $89.6 \%$ and $82.4 \%$, respectively, at a sLOX-1 cut-off value of $91.0 \mathrm{pg} / \mathrm{ml}$. Diagnostic sensitivity and specificity for NSTEMI were $79.5 \%$ and $82.4 \%$, respectively, at a sLOX-1 cut-off value of $90.3 \mathrm{pg} / \mathrm{ml}$. AMl, acute myocardial infarction. 


\begin{tabular}{lc|}
\hline Table 4. Characteristics of Patients in Study-2 & \\
Male & $\mathbf{n}(\%)$, mean \pm SD or median (25-75th percentiles) \\
Age (years) & $25(93)$ \\
Risk factors & $63 \pm 13$ \\
Familial history of myocardial infarction & \\
Hypertension & $3(11)$ \\
Diabetes & $16(59)$ \\
Dyslipidemia & $12(44)$ \\
Smoking & $20(74)$ \\
Time intervals (min) & $21(78)$ \\
Time from the onset of STEMI to ER & \\
Time from the onset of STEMI to start CAG & $89(66-173)$ \\
Time from the onset of STEMI to termination of PCI & $142(100-224)$ \\
\hline
\end{tabular}

$\mathrm{ER}$, emergency room; CAG, coronary angiography; $\mathrm{PCl}$, percutaneous coronary intervention. Other abbreviations see in Table 1.

A

$(\mathrm{pg} / \mathrm{mL})$

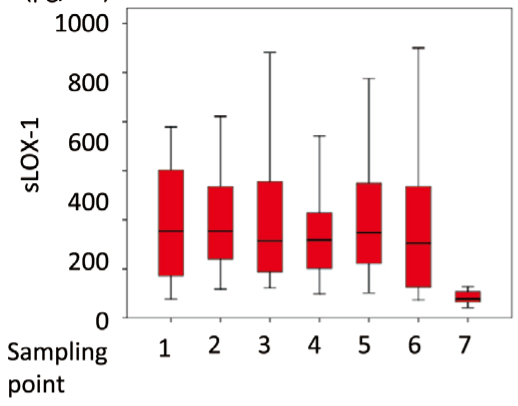

D

(ng/mL)

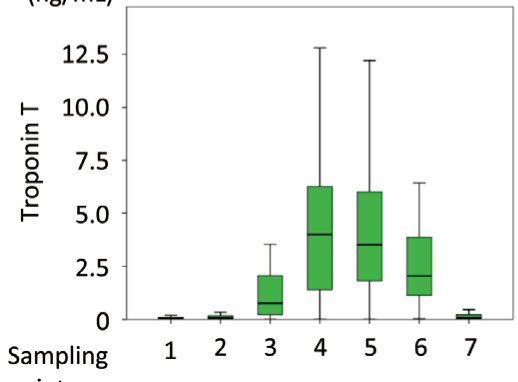

point
B

$(\mathrm{ng} / \mathrm{mL})$

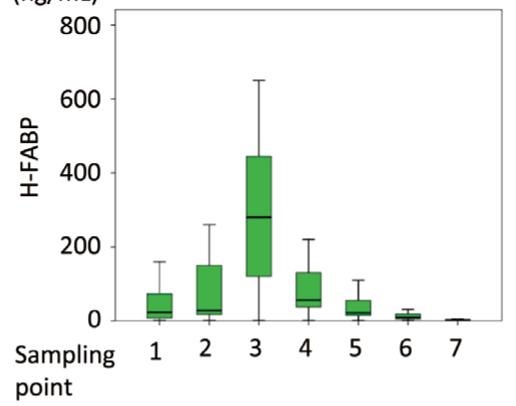

E

(ng/mL)

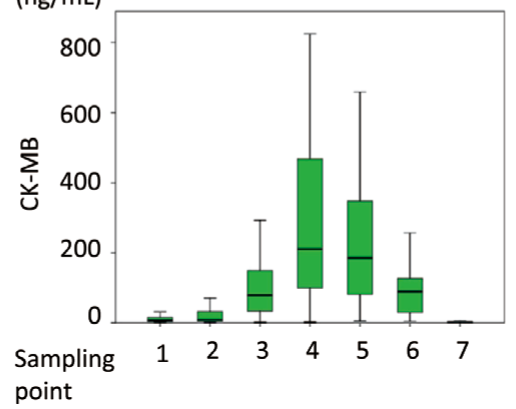

C

(ng/mL)

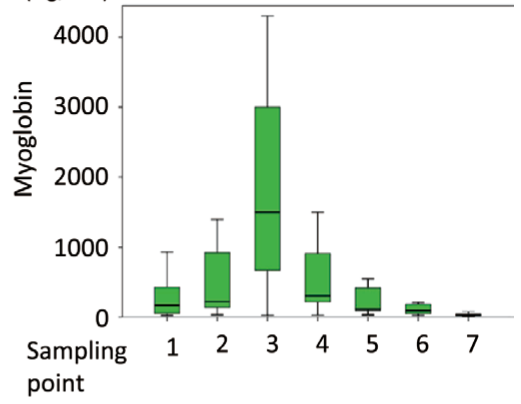

Figure 3. Time-dependent changes in soluble lectin-like oxidized low-density lipoprotein receptor-1 (sLOX-1) (A), heart-type fatty acid-binding protein (H-FABP) (B), myoglobin (C), troponin T (D), and creatine kinase-MB (CK-MB) (E). Center horizontal lines indicate median values. Lower and upper edges of boxes indicate 25th and 75th percentiles, and lower and upper bars indicate 10th and 90th percentiles. Sampling points 1 (upon emergency room (ER) arrival), 2 (before starting coronary angiography) and 3 (after termination of percutaneous coronary intervention) were 89, 142 and 230 min (median) after ST-elevation myocardial infarction (STEMI) onset, respectively. Sampling points 4, 5 and 6 were 6, 12 and 24h after arrival at ER, respectively. Sampling point 7 (at discharge from hospital) was 16 days (median) after the onset of STEMI.

\section{Discussion}

LOX-1, a receptor for atherogenic oxidized LDL, ${ }^{18}$ the expression of which is induced by factors related to atherogenesis and plaque vulnerability, such as proinflammatory cytokines, ${ }^{24}$ angiotensin II ${ }^{25}$ high glucose ${ }^{26}$ and oxidized LDL ${ }^{27}$, is expressed on the surface of intimal smooth muscle cells ${ }^{28}$ and lipid-laden macrophages ${ }^{28,29}$ in advanced human atherosclerotic plaques. LOX-1, furthermore, is cleaved at the membrane-proximal extracellular domain by proteases, including a disintegrin and metalloproteinase ${ }^{19,20}$ that may also be associated with plaque vulnerability or rupture, resulting in sLOX-1 release into the circulation. ${ }^{20}$ In experimental animal models, LOX-1 expression is closely associated with morpho- 


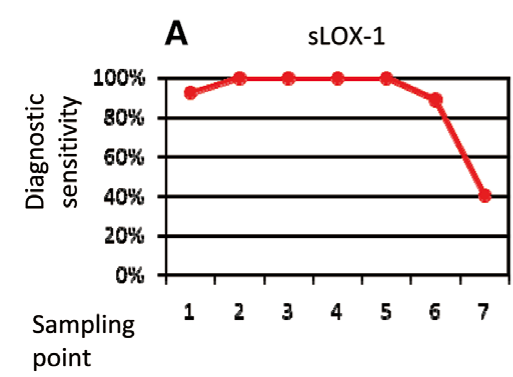

D Troponin T

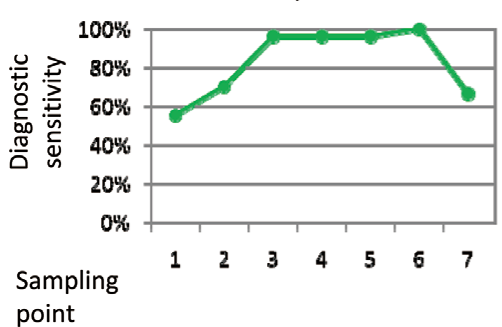

B

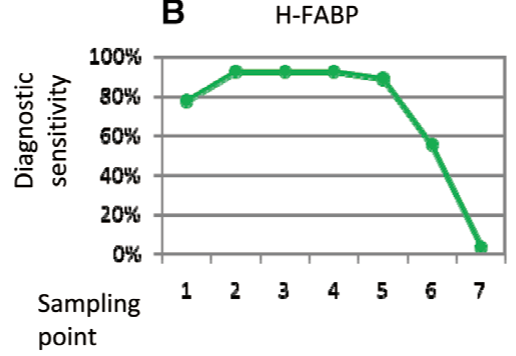

E

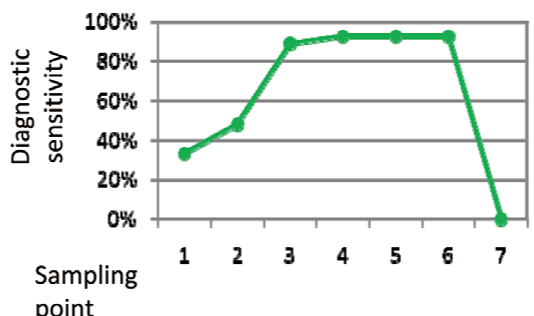

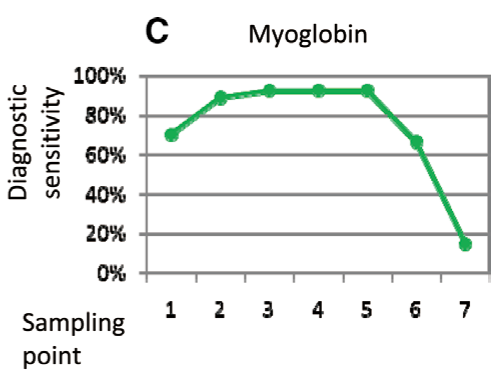

point

Figure 4. Time-dependent changes in the diagnostic sensitivity of the biomarkers for STEMI: sLOX-1 (A), H-FABP (B), myoglobin (C), troponin T (D), and CK-MB (E). The respective cut-off values were $91.0 \mathrm{pg} / \mathrm{ml}, 6.2 \mathrm{ng} / \mathrm{ml}, 60 \mathrm{ng} / \mathrm{ml}, 0.02 \mathrm{ng} / \mathrm{ml}$ and $10.0 \mathrm{ng} / \mathrm{ml}$. Abbreviations and sampling points are the same as those described in Figure 3.

logical plaque instability and cell apoptosis, as well as with the expression of matrix metalloproteinases and tissue factor, all of which are associated with plaque rupture and thrombus formation. ${ }^{30-32}$ Thus, proteases with enhanced activities in ruptured or rupture-prone vulnerable plaques may be responsible for the cleavage of LOX-1, which is abundantly expressed in advanced coronary atherosclerotic plaques. Taken together, these findings suggest that sLOX-1 is regarded as a biomarker of plaque rupture or plaque instability.

Because plaque instability or rupture precedes cardiac ischemia and necrosis in AMI, sLOX-1 release is expected to precede the release of biomarkers for myocardial injury or necrosis, such as CK-MB, cardiac troponins, myoglobin and $\mathrm{H}-\mathrm{FABP}$, in AMI. The results of the present study clearly demonstrate that plasma levels of sLOX-1 are useful for diagnosing AMI, especially the early stage. In this study, the plasma SLOX-1 level was elevated at the earliest stage $(89 \mathrm{~min}$ from the symptom onset) of STEMI, which was earlier than the elevation of cardiomyocyte injury or damage markers such as troponin T, H-FABP, myoglobin and CK-MB, reflecting plaque rupture or instability. In addition, plasma sLOX-1 levels were higher in males and smokers than in females and non-smokers, probably because estrogen and smoking affect plaque vulnerability by protecting vascular cells from inflammation (the former) ${ }^{33}$ and by inducing oxidative stress and inflammation (the latter). ${ }^{34}$ According to our multivariable logistic regression analysis including SLOX-1, male sex and smokers in Study-1, sLOX-1 is an independent factor associated with AMI (Table 3). In the Study-1 subjects, both the sLOX-1 and peak CK-MB levels were higher in STEMI than in NSTEMI patients, and sLOX-1 levels poorly, but significantly, correlated with peak CK-MB levels, suggesting that the amount of vulnerable plaque burden may be poorly, but significantly, associated with myocardial infarct size. In Study-2, on the other hand, plasma sLOX-1 levels fell almost to the levels of the control subjects at 16 days (median) after the onset of STEMI. These time-dependent reductions might result from the intensive treatment after hospitalization, including the pharmacological effects of statins, angiotensinconverting enzyme inhibitors, angiotensin-receptor blockers, and antiplatelet drugs.

The World Health Organization states that a clinical diagnosis of AMI is usually based on a history of chest pain, ECG changes and the elevation of cardiospecific enzymes. ${ }^{35}$ Although CK-MB is the gold standard for a diagnosis of AMI and we have extensive clinical experience, it is not a sensitive marker of increased myocardial necrosis. ${ }^{6}$ Many clinical studies during the 1990 s clarified the values of troponins I and $\mathrm{T}$ for the diagnosis and risk stratification of AMI. ${ }^{7-11}$ Consequently, the European Society of Cardiology and the American College of Cardiology Committee jointly revised the definition of AMI, ${ }^{15}$ and cardiac troponins are listed as the biomarkers of preference in the latest definition, ${ }^{16}$ which states that AMI is the diagnosis when blood levels of cardiac biomarkers, preferably troponin I or T, are increased in the clinical setting of acute myocardial ischemia. However, the sensitivity of CK-MB and troponin (I or T) within $4 \mathrm{~h}$ of symptom onset (early stage of AMI) is low because they only start to elevate 3-4h after onset. On the other hand, myoglobin and H-FABP serve as adjunct modalities for a diagnosis of early stage AMI because levels of these biomarkers begin to increase $1 \mathrm{~h}$ after onset. ${ }^{13}$ However, these biomarkers are not highly specific for a diagnosis of AMI, and combination with other modalities is recommended to diagnose the early stage of AMI. ${ }^{12} \mathrm{We}$, therefore, speculate that the diagnostic sensitivity and specificity for AMI in the early stage would be improved by combination of biomarkers reflecting cardiomyocyte injury or damage and those for plaque rupture or vulnerability, including sLOX-1. It would be desirable if rapid measurement of blood sLOX-1 levels in the ER became commercially available in the future. 


\section{Conclusion}

sLOX-1, a biomarker for plaque rupture or vulnerability, but not for myocardial damage, is more useful for diagnosing STEMI in the earliest stage than biomarkers of myocardial damage. However, the limitation of the present study would be the relatively small sample size, and thus the present findings should be confirmed by multicenter studies with larger sample sizes including NSTEMI patients. In addition, a recent pilot study has shown that SLOX-1 predicted prognosis, such as future recurrence of ACS or death, in ACS patients, ${ }^{36}$ which also should be confirmed by large-scale prospective studies in the future.

\section{Acknowledgments}

We thank the medical and nursing staff of Nippon Medical School Chiba Hokusoh Hospital, and the patients for participating in this study.

\section{References}

1. Ross J Jr. A 50-year research journey: From laboratory to clinic. Circ J 2009; 73: 3-12.

2. Lloyd-Jones D, Adams R, Carnethon M, De Simone G, Ferguson TB, Flegal K, et al. Heart disease and stroke statistics-2009 update: A report from the American Heart Association Statistics Committee and Stroke Statistics Subcommittee. Circulation 2009; 119: e21-e181.

3. De Luca G, Suryapranata H, Ottervanger JP, Antman EM. Time delay to treatment and mortality in primary angioplasty for acute myocardial infarction: Every minute of delay counts. Circulation 2004; 109: 1223-1225.

4. Boden WE, Eagle K, Granger CB. Reperfusion strategies in acute ST-segment elevation myocardial infarction: A comprehensive review of contemporary management options. J Am Coll Cardiol 2007; 50: $917-929$.

5. Yasuda S, Shimokawa H. Acute myocardial infarction: The enduring challenge for cardiac protection and survival. Circ J 2009; 73: 2000-2008.

6. Lee TH, Goldman L. Serum enzyme assays in the diagnosis of acute myocardial infarction: Recommendations based on a quantitative analysis. Ann Intern Med 1986; 105: 221-233.

7. Antman EM, Grudzien C, Sacks DB. Evaluation of a rapid bedside assay for detection of serum cardiac troponin T. JAMA 1995; 273: $1279-1282$.

8. Ohman EM, Armstrong PW, Christenson RH, Granger CB, Katus $\mathrm{HA}$, Hamm CW, et al. Cardiac troponin T levels for risk stratification in acute myocardial ischemia: GUSTO IIA Investigators. $N$ Engl J Med 1996; 335: 1333-1341.

9. Antman EM, Tanasijevic MJ, Thompson B, Schactman M, McCabe $\mathrm{CH}$, Cannon $\mathrm{CP}$, et al. Cardiac-specific troponin I levels to predict the risk of mortality in patients with acute coronary syndromes. N Engl J Med 1996; 335: $1342-1349$.

10. Hamm CW, Goldmann BU, Heeschen C, Kreymann G, Berger J, Meinertz T. Emergency room triage of patients with acute chest pain by means of rapid testing for cardiac troponin $\mathrm{T}$ or troponin $\mathrm{I}$. N Engl J Med 1997; 337: 1648-1653.

11. Antman EM, Sacks DB, Rifai N, McCabe CH, Cannon CP, Braunwald E. Time to positivity of a rapid bedside assay for cardiacspecific troponin $\mathrm{T}$ predicts prognosis in acute coronary syndromes: A Thrombolysis in Myocardial Infarction (TIMI) 11A substudy. J Am Coll Cardiol 1998; 31: 326-330.

12. Jurlander B, Clemmensen P, Wagner GS, Grande P. Very early diagnosis and risk stratification of patients admitted with suspected acute myocardial infarction by the combined evaluation of a single serum value of cardiac troponin-T, myoglobin, and creatine kinase MB(mass). Eur Heart J 2000; 21: 382 - 389.

13. Seino Y, Ogata K, Takano T, Ishii J, Hishida H, Morita $H$, et al. Use of a whole blood rapid panel test for heart-type fatty acid-binding protein in patients with acute chest pain: Comparison with rapid troponin T and myoglobin tests. Am J Med 2003; 115: 185-190.

14. Morrow DA, Cannon CP, Jesse RL, Newby LK, Ravkilde J, Storrow $\mathrm{AB}$, et al. National Academy of Clinical Biochemistry Laboratory Medicine Practice Guidelines: Clinical characteristics and utilization of biochemical markers in acute coronary syndromes. Circulation 2007; 115: e $356-\mathrm{e} 375$.

15. Myocardial infarction redefined: A consensus document of The Joint
European Society of Cardiology/American College of Cardiology Committee for the redefinition of myocardial infarction. Eur Heart $J$ 2000; 21: 1502-1513.

16. Thygesen K, Alpert JS, White HD, Joint ESCA/AHA/WHF Joint Task Force for the Redefinition of Myocardial Infarction. Universal definition of myocardial infarction. Eur Heart J 2007; 28: $2525-2538$.

17. Libby P. Current concepts of the pathogenesis of the acute coronary syndromes. Circulation 2001; 104: $365-372$.

18. Sawamura T, Kume N, Aoyama T, Moriwaki H, Hoshikawa H, Aiba Y, et al. An endothelial receptor for oxidized low-density lipoprotein. Nature 1997; 386: 73-77.

19. Mitsuoka H, Kume N, Hayashida K, Inui-Hayashiada A, Aramaki Y, Toyohara M, et al. Interleukin 18 stimulates release of soluble lectin-like oxidized LDL receptor-1 (sLOX-1). Atherosclerosis 2009; 202: $176-182$.

20. Murase T, Kume N, Kataoka H, Minami M, Sawamura T, Masaki $\mathrm{T}$, et al. Identification of soluble forms of lectin-like oxidized LDL receptor-1. Arterioscler Thromb Vasc Biol 2000; 20: 715-720.

21. Ueda A, Kume N, Hayashida K, Inui-Hayashida A, Asai M, Kita T, et al. ELISA for soluble form of lectin-like oxidized LDL receptor1, a novel marker of acute coronary syndrome. Clin Chem 2006; 52: $1210-1211$.

22. Hayashida K, Kume N, Murase T, Minami M, Nakagawa D, Inada $\mathrm{T}$, et al. Serum soluble lectin-like oxidized low-density lipoprotein receptor-1 levels are elevated in acute coronary syndrome: A novel marker for early diagnosis. Circulation 2005; 112: 812-818.

23. Nakamura M, Ohta H, Kume N, Hayashida K, Tanaka M, Mitsuoka $\mathrm{H}$, et al. Generation of monoclonal antibodies against a soluble form of lectin-like oxidized low-density lipoprotein receptor-1 and development of a sensitive chemiluminescent enzyme immunoassay. J Pharmacol Biomed Anal 2010; 51: 158-163.

24. Kume N, Murase T, Moriwaki H, Aoyama T, Sawamura T, Masaki $\mathrm{T}$, et al. Inducible expression of lectin-like oxidized LDL receptor-1 in vascular endothelial cells. Circ Res 1998; 83: 322-327.

25. Li DY, Zhang YC, Philips MI, Sawamura T, Mehta JL. Upregulation of endothelial receptor for oxidized low-density lipoprotein (LOX-1) in cultured human coronary artery endothelial cells by angiotensin II type 1 receptor activation. Circ Res 1999; 84: 1043-1049.

26. Li L, Sawamura T, Renier G. Glucose enhances endothelial LOX-1 expression: Role for LOX-1 in glucose-induced human monocyte adhesion to endothelium. Diabetes 2003; 52: 1843-1850.

27. Kataoka H, Kume N, Miyamoto S, Minami M, Morimoto M, Hayashida K, et al. Oxidized LDL modulates Bax/Bcl-2 through the lectinlike Ox-LDL receptor-1 in vascular smooth muscle cells. Arterioscler Thromb Vasc Biol 2001; 21: 955-960.

28. Moriwaki H, Kume N, Kataoka H, Murase T, Nishi E, Sawamura $\mathrm{T}$, et al. Expression of lectin-like oxidized low density lipoprotein receptor-1 in human and murine macrophages: Upregulated expression by TNF-alpha. FEBS Lett 1998; 440: 29-32.

29. Kataoka H, Kume N, Miyamoto S, Minami M, Moriwaki H, Murase $\mathrm{T}$, et al. Expression of lectinlike oxidized low-density lipoprotein receptor-1 in human atherosclerotic lesions. Circulation 1999; 99: $3110-3117$

30. Ishino S, Mukai T, Kume N, Asano D, Ogawa M, Kuge Y, et al. Lectin-like oxidized LDL receptor-1 (LOX-1) expression is associated with atherosclerotic plaque instability: Analysis in hypercholesterolemic rabbits. Atherosclerosis 2007; 195: 48-56.

31. Kuge Y, Kume N, Ishino S, Takai N, Ogawa Y, Mukai T, et al. Prominent lectin-like oxidized low density lipoprotein (LDL) receptor-1 (LOX-1) expression in atherosclerotic lesions is associated with tissue factor expression and apoptosis in hypercholesterolemic rabbits. Biol Pharmacol Bull 2008; 31: 1475-1482.

32. Ishino S, Mukai T, Kuge Y, Kume N, Ogawa M, Takai N, et al. Targeting of lectinlike oxidized low-density lipoprotein receptor 1 (LOX-1) with 99mTc-labeled anti-LOX-1 antibody: Potential agent for imaging of vulnerable plaque. J Nucl Med 2008; 49: $1677-$ 1685 .

33. Mendelsohn ME, Karas RH. The protective effects of estrogen on the cardiovascular system. N Engl J Med 1999; 340: 1801 - 1811.

34. Yanbaeva DG, Dentener MA, Creutzberg EC, Wesseling G, Wouters EF. Systemic effects of smoking. Chest 2007; 131: 1557-1566.

35. Nomenclature and criteria for diagnosis of ischemic heart disease. Report of the Joint International Society and Federation of Cardiology/World Health Organization task force on standardization of clinical nomenclature. Circulation 1979; 59: 607-609.

36. Kume N, Mitsuoka H, Hayashida K, Tanaka M, Kita T. Soluble lectin-like oxidized low-density lipoprotein receptor-1 predicts prognosis after acute coronary syndrome: A pilot study. Circ J 2010; 74: $1399-1404$. 